01,11

\title{
Электронная структура и магнитный фазовый переход в MnSi
}

\author{
(C) А.А. Повзнер, А.Г. Волков, Т.А. Ноговицына \\ Уральский федеральный университет им. Б.Н. Ельцина, \\ Екатеринбург, Россия \\ E-mail: a.a.povzner@urfu.ru
}

(Поступила в Редакцию 27 декабря 2016 г.)

В рамках модели электронной структуры, следующей из ab initio LDA $+U+\mathrm{SO}$-расчетов, описаны температурные изменения амплитуды нулевых и тепловых спиновых флуктуаций в геликоидальном ферромагнетике MnSi. Получено, что возникающее при температуре $T_{S}$ (лежащей в окрестности магнитного фазового перехода) резкое уменьшение амплитуды нулевых спиновых флуктуаций ведет к неустойчивости ферромагнитных решений (смена знака параметра межмодового взаимодействия). Это же является причиной наблюдаемых магнитообъемного эффекта и резкого изменения радиуса спиновых корреляций. Результаты расчета объемного коэффициента теплового расширения хорошо согласуются с наблюдаемой аномалией в области магнитного фазового перехода.

DOI: 10.21883/FTT.2017.07.44584.458

1. Кристаллическая структура сильно коррелированных $\mathrm{MnSi}, \mathrm{Fe}_{1-x} \mathrm{Co}_{x} \mathrm{Si}, \mathrm{Fe}_{1-y} \mathrm{Mn}_{y} \mathrm{Si}$ и др. относится к структурному типу $B 20$ с пространственной группой $P 2{ }_{1} 3$, для которой характерно отсутствие центра инверсии $[1,2]$. Такая симметрия обусловливает возникновение энергетической щели в спектре $d$-электронов [3] и приводит к возникновению антисимметричного релятивистского обмена Дзялошинского-Мории (ДМ) [4,5]. При этом в рассматриваемой сильно коррелированной электронной системе формируется геликоидальное упорядочение с аномально большими магнитными периодами (порядка 100-1000 А) [6-8], вследствие чего описание электронной структуры сталкивается с заметными трудностями учета длиннопериодической спиновой подсистемы $\left(\mathbf{M}_{\mathbf{q}_{0}} \neq 0\right)$. Поэтому в $a b$ initio $\mathrm{LDA}+U+\mathrm{SO}$-расчетах электронной структур [3,9-11] пренебрегают ДМ-взаимодействием и получают основное ферромагнитное состояние для $\mathrm{MnSi}$. Модельный учет в рассматриваемом ферромагнитном основном состоянии ДМ-взаимодействия приводит к представлениям о ферромагнитном геликоиде, описываемом моделью Янсена-Бака [6,7].

Вместе с тем в области от $T_{S}$ (температуры резкого уменьшения амплитуды нулевых спиновых флуктуаций) до $T_{C}$ (температуры перехода в парамагнитное coстояние) экспериментально обнаружено возникновение вращения волнового вектора геликоидальной структуры, которое указывает на исчезновение фиксированной оси квантования геликоидальной спирали („катастрофа“ модели Янсена-Бака) [12]. При этом возникает резкое уменьшение радиуса спиновых корреляций (который сохраняет конечное значение при $T_{C}$ ), а также формируются лямбдаподобные аномалии теплоемкости и коэффициента объемного теплового расширения (КТР) [2,7]. Однако причины изменения магнитного состояния в области магнитного перехода, особенностей температурной зависимости отрицательного КТР, а также роль уменьшения объема в формировании электронной структуры не выяснены.

2. В настоящей работе на основе спин-флуктуационной теории моделируется электронная структура и исследуются термодинамические свойства $\mathrm{MnSi}$ в окрестности магнитного перехода. Рассматривается сильно коррелированная электронная система с гамильтонианом, учитывающим энергию зонного движения в приближении $\mathrm{LDA}+U+\mathrm{SO}$ [3], внутриатомные кулоновские спиновые и зарядовые корреляции, а также антисимметричное релятивистское взаимодействие Дзялошинского-Мории. Слагаемые кулоновского взаимодействия представлены через спиновые и зарядовые операторы

$$
H=H_{0}+H_{U}+H_{\mathrm{DM}} .
$$

Здесь $H_{0}=\sum_{\mathbf{k}, m, \sigma} \varepsilon_{\mathbf{k}, m, \sigma}^{(\mathrm{LDA})} a_{\mathbf{k}, m, \sigma}^{+} a_{\mathbf{k}, m, \sigma}-$ диагонализованный в приближении $\mathrm{LDA}+U+\mathrm{SO}$ гамильтониан $d$-электронов, $a_{\mathbf{k}, m, \sigma}^{+}\left(a_{\mathbf{k}, m, \sigma}\right)-$ оператор рождения (уничтожения) электрона, $\mathbf{k}$ - вектор квазиимпульса, $m-$ индекс $d$-орбитали (см. работу [3] и ссылки в ней), $\sigma= \pm 1-$ спиновый индекс, $\varepsilon_{\mathbf{k}, m, \sigma}^{(\mathrm{LDA})}=\varepsilon_{\mathbf{k}, m}^{(\mathrm{LDA})}+\sigma \Delta-$ электронный спектр $d$-электронов, рассчитанный в приближении $\mathrm{LDA}+U+\mathrm{SO}$ (спектр $\varepsilon_{\mathbf{k}, m}^{(\mathrm{LDA})}$ образует для каждого значения $m$ две подзоны, разделенные щелью порядка $0.12 \mathrm{eV}$ [3]), $\Delta-$ спиновое расщепление электронных энергий (не зависящее от $m[3]$ ),

$$
\begin{aligned}
H_{U}= & \frac{1}{4} \sum_{q}\left[(U-J / 2)\left|\delta n_{q}\right|^{2}-(U+J) \sum_{m}\left|\delta n_{q, m}\right|^{2}\right] \\
& -\sum_{q}\left[J\left|\delta S_{q}^{z}\right|^{2}+(U-J) \sum_{m}\left|\delta S_{q, m}^{z}\right|^{2}\right]
\end{aligned}
$$

- поправка к приближению $\mathrm{LDA}+U+\mathrm{SO}$, учитывающая многочастичные хаббардовские корреляции, $J$ и $U$ - параметры хаббардовского и хундовского 
взаимодействий, $\delta n_{\mathbf{q}}=\sum_{m} \delta n_{\mathbf{q}, m}, \delta S=\sum_{m} \delta S_{\mathbf{q}, m}^{(z)}, \delta n_{\mathbf{q}, m}=$ $=\sum_{\sigma} n_{\mathbf{q}, m, \sigma}-\delta n_{q, 0} N_{m}^{(\mathrm{LDA})}, n_{\mathbf{q}, m, \sigma}=a_{\mathbf{k}, m, \sigma}^{+} a_{\mathbf{k}+\mathbf{q}, m, \sigma}, \quad \delta S_{\mathbf{q}, m}^{(z)}=$ $=S_{\mathbf{q} m}^{(z)}-\delta_{q, 0} M_{m}^{(\mathrm{LDA})}, \quad S_{\mathbf{q} m}^{(z)}=\sum_{\sigma} \sigma n_{\mathbf{q}, m, \sigma} / 2, \quad$ а $\quad M_{m}^{(\mathrm{LDA})} \quad$ и $N_{m}^{(\mathrm{LDA})}-$ средние в базисе $\mathrm{LDA}+U+\mathrm{SO}$ значения Фурье-образов операторов спиновой и зарядовой плотностей. Ввиду малости ДМ-взаимодействия по сравнению с другими обменными взаимодействиями ограничимся его учетом в приближении среднего поля

$$
H_{\mathrm{DM}} \approx 2 \sum_{m}\left(\mathbf{h}_{\mathbf{q}_{0}, m}^{(D)} \mathbf{S}_{\mathbf{q}_{0}, m}-\mathbf{d}_{\mathbf{q}_{0}}\left[\mathbf{M}_{\mathbf{q}_{0}, m} \times \mathbf{M}_{-\mathbf{q}_{0}, m}\right]\right),
$$

где $\mathbf{d}_{\mathbf{q}}=i d \mathbf{q}, d-$ константа Дзялошинского, $\mathbf{q}-$ вектор квазиимпульса, $\mathbf{M}_{\mathbf{q} 0, m}=\left\langle\mathbf{S}_{\mathbf{q}_{0}, m}\right\rangle-$ вектор неоднородной намагниченности, $\mathbf{h}_{\mathbf{q}_{0}}^{(D)}=\left[\mathbf{M}_{\mathbf{q}_{0}, m} \times \mathbf{d}_{-\mathbf{q}_{0}}\right]-$ среднее поле Дзялошинского, $\mathbf{S}_{\mathbf{q}}=\sum_{\sigma, \sigma^{\prime}} a_{\mathbf{k}, m, \sigma}^{+} \boldsymbol{\sigma}_{\sigma, \sigma^{\prime}} a_{\mathbf{k}+\mathbf{q}, m, \sigma^{\prime}}-$ Фурье-образ оператора спиновой плотности на узле, $\boldsymbol{\sigma}_{\sigma, \sigma^{\prime}}$ - вектор матриц Паули.

Свободную энергию электронной системы, описываемую гамильтонианом (1), будем определять, используя формализм преобразований Стратоновича-Хаббарда, сводящих многочастичные взаимодействия в (2) к взаимодействию электронов с флуктуирующими обменными $(\xi)$ и зарядовыми $(\eta)$ полями

$$
\begin{aligned}
& F=-T \ln Z=-T \ln \int_{-\infty}^{\infty}(d \xi, d \eta) Z(\xi, \eta) \exp \left\{-\sum_{q, m}\left|\eta_{q, m}\right|^{2}\right. \\
& \left.-b\left|\sum_{q, m} \eta_{q, l, m}\right|^{2}-(i c / T) \sum_{m} \eta_{0, m} N_{m}^{(\mathrm{LDA})}\right\} \exp \left\{-a\left|\sum_{q, m} \xi_{q, m}\right|^{2}\right. \\
& \left.-\sum_{q, m}\left|\xi_{q, m}-\mathbf{h}_{q, m}^{(D)} / c-\delta_{q, 0} \Delta / c\right|^{2}-2(c / T) \sum_{m} M_{m}^{(\mathrm{LDA})} \xi_{0, m}^{(z)}\right\},
\end{aligned}
$$

где $T-$ температура в энергетических единицах, $\quad a=J U(U-J)^{-1}(U+5 J)^{-1}, \quad b=4 U(U-5 J)^{-1}$, $c=U^{1 / 2} T^{1 / 2}, Z(\xi, \eta)=\operatorname{Sp} T_{\tau} \exp \left(-H_{\mathrm{eff}}(\xi, \eta) / T\right)-$ статистическая сумма электронов, движущихся в одной из конфигураций стохастических обменных $(\xi)$ и зарядовых $(\eta)$ полей,

$$
\begin{aligned}
H_{\mathrm{eff}}= & \sum_{k, m, \sigma} \varepsilon_{\mathbf{k}, m}^{(\mathrm{LDA})} a_{k, m, \sigma}^{+} a_{k, m, \sigma}+\sum_{q, m}\left[(i c / 2) \eta_{-q, m} n_{q, m}\right. \\
& \left.+c \xi_{-q, m} \mathbf{S}_{q, m}\right]-\sum_{m} \mathbf{d}_{\mathbf{q}_{0}}\left[\mathbf{M}_{\mathbf{q}_{0}, m} \times \mathbf{M}_{-\mathbf{q}_{0}, m}\right]
\end{aligned}
$$

- эффективный гамильтониан,

$$
\begin{aligned}
(d \eta d \xi)= & {\left[(U-J) U^{-1} \prod_{m} d \xi_{0, m} d \eta_{0, m}\right] } \\
& \times\left[\prod_{q \neq 0, m, j} \prod_{m} d \xi_{q, m}^{(j)} d \eta_{q, m}^{(j)}\right] .
\end{aligned}
$$

Квантово-статистическое усреднение в выражениях статистической суммы в рассматриваемой задаче о фазовых переходах в киральных магнетиках с аномально большими периодами магнитной спиновой структуры можно выполнить на основе приближения однородных локальных полей Герца-Кленина [13]. В рамках этого приближения учитывается пространственно-временна́я неоднородность вершинных частей второго порядка, к которым сводится фактор обменного усиления магнитной восприимчивости, аномально сильно зависящий от $\mathbf{q}$ и $\omega$ в длиннопериодических структурах и вблизи $T_{C}$.

Анализ выражения для статистической суммы показывает, что имеется связь между значениями полей $\xi$, определяемыми условиями седловой точки (4), с намагниченностями $M_{0, m}=U^{-1}\left(c \xi_{0, m}^{(z)}-U M_{m}^{(\mathrm{LDA})}\right)$ и $\mathbf{M}_{\mathbf{q}_{0}, m}=U^{-1}\left(c \xi_{\mathbf{q}_{0}, m}-\mathbf{h}_{\mathbf{q}_{0}, m}\right)$ на векторах $\mathbf{q}=0$ и $\pm \mathbf{q}_{0}$ соответственно, а также с парными спиновыми корреляторами $\left\langle\mathbf{S}_{q, m} \mathbf{S}_{-q, m}\right\rangle=(c / U)^{2}\left(2\left|\xi_{q, m}\right|^{2}-1\right)$ и среднеквадратичным магнитным моментом на узле $M_{m}=\left(M_{0, m}^{2}+\left|\mathbf{M}_{\mathbf{q}_{0}, m}\right|^{2}+\left\langle\mathbf{M}_{m}^{2}\right\rangle\right)^{1 / 2}$, определяемым значениями намагниченностей и амплитуды спиновых флуктуаций. В соответствии с условиями седловой точки (4)

$$
\begin{aligned}
& \left\langle M_{m}^{2}\right\rangle=(2 \pi U)^{-1} \sum_{\mathbf{q}, \gamma} \int_{0}^{\infty}\left(1+2 f_{\mathrm{B}}(\omega / T)\right) \\
& \quad \times \operatorname{Im}\left(D_{m}^{-1}+2 \kappa M_{0, m}^{2} \delta_{\gamma, z}+2 \kappa_{m}\left|M_{\mathbf{q}_{0}, m}^{(\gamma)}\right|+X_{q}\right)^{-1} d \omega,
\end{aligned}
$$

$f_{\mathrm{B}}(\omega, T)-$ функция Бозе, $\left\langle\mathbf{M}_{m}^{2}\right\rangle=\left\langle\mathbf{M}_{m}^{2}\right\rangle_{0}+\left\langle\mathbf{M}_{m}^{2}\right\rangle_{T}$, $\left\langle\mathbf{M}_{m}^{2}\right\rangle_{0}$ и $\left\langle\mathbf{M}_{m}^{2}\right\rangle_{T}$ - амплитуды нулевых и тепловых флуктуаций спиновой плотности электронов, причем суммируя (6) получаем флуктуационно-диссипативную теорему. Здесь $D_{m}^{-1}=1-\chi_{\perp}^{(m)}+\kappa\left\langle\mathbf{M}_{m}^{2}\right\rangle / 3-$ фактор обменного усиления парамагнитной восприимчивости,

$$
\begin{gathered}
\chi_{\perp}^{(m)}=\left(2 U M_{m}\right)^{-1} \sum_{\alpha= \pm 1} \alpha \int_{-\infty}^{\infty} g_{\alpha, m}(\varepsilon) f(\varepsilon-\mu) d \varepsilon, \\
\chi_{\|}^{(m)}=2\left(\sum_{\alpha= \pm 1} g_{\alpha, m}(\mu)\right)^{-1} \prod_{\alpha= \pm 1} g_{\alpha, m}(\mu), \\
\kappa_{m}=\left(U^{3} / M_{m}^{2}\right)\left[\chi_{\perp}^{(m)}-\chi_{\|}^{(m)}\right]
\end{gathered}
$$

- коэффициент межмодовой связи, определяемый плотностью состояний (см. (7)) спин-симметризованных и спин-антисимметризованных электронных состояний, $f(\varepsilon-\mu)-$ функция Ферми-Дирака, $\mu-$ химический потенциал, определяемый из условия электронейтральности: $N=-\partial F / \partial \mu$. При этом в соответствии с известной аппроксимацией функции Линдхарда [14]

$$
\begin{aligned}
X(\mathbf{q}, \omega)= & \left(A\left(\mathbf{q} / k_{\mathrm{F}}\right)^{2}-i B U^{-1} \frac{\omega}{\left|\mathbf{q} / k_{\mathrm{F}}\right|} \theta\left(T_{0}\left|\mathbf{q} / K_{\mathrm{F}}\right|-\omega\right)\right) \\
& \times \theta\left(2 k_{\mathrm{F}}-q\right),
\end{aligned}
$$

где коэффициенты $A$ и $B$ пропорциональны плотности электронных состояний на уровне Ферми, $T_{0}=V_{\mathrm{F}} k_{\mathrm{F}}$, 
$V_{\mathrm{F}}-$ скорость на поверхности Ферми, $k_{\mathrm{F}}-$ модуль вектора Ферми, $\theta(x)-\theta$-функция. ${ }^{1}$

В рамках метода производящего функционала (в результате дифференцирования (4) по $\varepsilon_{\mathbf{k}, m}^{(\mathrm{LDA})}$ ) можно определить плотность электронных состояний, которая оказывается перенормированной флуктуациями спиновой плотности и представляется в виде суперпозиции спин-симметризованных $(\alpha=+1)$ и спин-антисимметризованных $(\alpha=-1)$ плотностей электронных состояний сильно коррелированных электронов, причем спиновые флуктуации ведут к перемешиванию состояний с разными значениями полного орбитального магнитного момента.

Учитывая возникающие в рассматриваемом киральном магнетике магнитообъемные эффекты, будем рассматривать плотности электронных состояний, содержащие зависимости от объема. Воспользуемся для плотности электронных состояний моделью соответственных состояний $g(\varepsilon)=\left(W / W_{0}\right)^{-1} g(\varepsilon)$; считая в соответствии с [15], что относительная ширина зоны $\left(W / W_{0}\right) \sim\left(V / V_{0}\right)^{-n / 3} \quad\left(n=5\right.$ для $d$-электронов, $V_{0}$ и $W_{0}$ - объем кристалла и ширина зоны в отсутствие теплового расширения), имеем

$$
\begin{aligned}
g_{m, \alpha}(\varepsilon)= & 2^{-1} W^{-1} \sum_{m, \sigma= \pm 1}\left(1-\sigma \alpha M_{0, m} / M_{m}\right) \\
& \times g_{0}^{(m, \sigma)}\left(\varepsilon+\alpha \frac{U M_{m}}{W / W_{0}}\right) .
\end{aligned}
$$

Выражение для свободной энергии при этом будет содержать вклад упругой деформации $F \rightarrow F_{\mathrm{el}}-K \Delta V^{2} / 2$, где $K$ - изотермическая сжимаемость, причем электронная часть записывается в виде

$$
F_{\mathrm{el}}=-T \ln Z=F_{\mathrm{ex}}+F_{0 \mathrm{el}}+F_{\mathrm{sf}},
$$

где

$$
\begin{aligned}
& F_{\mathrm{ex}}=\sum_{q\left(\neq \mathbf{q}_{0}\right), m, \gamma} U\left(1+\left(W_{0} / W\right) X_{q}\right)\left\langle\mathbf{M}_{q, m, \gamma}^{2}\right\rangle \\
& +\sum_{m}\left\{U\left(1+\left(W_{0} / W\right) X_{\mathbf{q}_{0}}\right)\left|\mathbf{M}_{\mathbf{q}_{0}, m}\right|^{2}+2 d_{\mathbf{q}_{0}}\left[\mathbf{M}_{\mathbf{q}_{0}, m} \mathbf{M}_{-\mathbf{q}_{0}, m}\right]\right\}
\end{aligned}
$$

- энергия хаббардовского обменного взаимодействия спиновых магнитных моментов,

$$
F_{0 \mathrm{el}}=T \sum_{\alpha, m} \int g_{m, \alpha}(\varepsilon) \ln \left(1+\exp \left(\frac{\mu-\varepsilon}{T}\right)\right) d \varepsilon-\mu N
$$

— одноэлектронный вклад в свободную энергию,

$$
\begin{aligned}
F_{\mathrm{sf}}= & (T / 2) \sum_{q\left(\neq \mathbf{q}_{0}\right), \gamma, m} \ln \left(D_{m}^{-1}+2 \kappa M_{0, m}^{2} \delta_{\gamma, z}\right. \\
& \left.+2 \kappa_{m}\left|M_{\mathbf{q}_{0}, m}^{(\gamma)}\right|+\left(W_{0} / W\right) X_{q}\right)
\end{aligned}
$$

- свободная энергия спиновых флуктуаций.

\footnotetext{
${ }^{1}$ В рассматриваемой электронной структуре $\mathrm{MnSi}[3]$ зависимостью $X(\mathbf{q}, \omega)$ от индекса $m$ можно пренебречь.
}

Уравнения магнитного состояния, получаемые из условий минимума (8) по однородной и неоднородной намагниченности, содержат эффекты, связанные с флуктуациями модуля магнитного момента и чисел заполнения на узле, и имеют вид

$$
\begin{aligned}
\mathbf{M}_{\mathbf{q}_{0}, m}\left(D_{m}^{-1}\right. & \left.+X\left(\mathbf{q}_{0}, 0\right)+a\right) \\
& +\frac{1}{2} \kappa \mathbf{M}_{-\mathbf{q}_{0}, m}\left(\mathbf{M}_{\mathbf{q}_{0}, m}\right)^{2}=\mathbf{h}_{-\mathbf{q}_{0}, m}^{(D)}, \\
M_{0, m}\left(D_{m}^{-1}+\right. & \left.\kappa_{m}\left|\mathbf{M}_{\mathbf{q}_{0}, m}\right|^{2}+a\right) \\
= & -\Delta\left(D_{m}^{-1}+a-\kappa_{m}\left\langle\mathbf{M}_{m}^{2}\right\rangle / 3\right),
\end{aligned}
$$

где

$$
\mathbf{M}_{\mathbf{q}_{0}, m}=\mathbf{M}_{-\mathbf{q}_{0}, m}^{*}=2^{-1 / 2}\left(\mathbf{i} M_{q_{0}, m}+\mathbf{j} M_{q_{0}, m}\right),
$$

i и $\mathbf{j}$ - орты, лежащие в геликоидальной плоскости. Уравнения (9), (10) при $\kappa_{m}>0$ соответствуют модели Янсена-Бака [6] для ферромагнитного геликоида, а при $\kappa_{m}<0$ ферромагнитные решения становятся неустойчивыми.

В свою очередь модуль вектора гелимагнитного упорядочения $\left(\mathbf{q}_{0}\right)$, который определялся из условия максимума модуля неоднородной намагниченности, удовлетворяет равенству $q_{0} \approx d / 2 U A$.

Совместный анализ выражений (6)-(10) позволяет с учетом результатов $\mathrm{LDA}+U+\mathrm{SO}$-расчетов получить возможную модель плотности электронных состояний, учитывающую наряду с кулоновскими взаимодействиями, антисимметричный релятивистский обмен Дзялошинского-Мории. Известная из $\mathrm{LDA}+U+\mathrm{SO}-$ расчетов спиновая система является ферромагнитной, а электронная структура представляет собой расщепленные по направлениям спина спин-поляризованные подзоны с раздвижкой, пропорциональной намагниченности [3,9-11]. Однако учет ДМ-взаимодействия „закручивает“" спины, и однородная намагниченность должна исчезать. Анализ (6) - (10) показывает, что в этом случае $\left(M_{0, m}=0\right)$ и при $\Delta \neq 0$ раздвижка спин-поляризованных подзон исчезает. При этом должен существовать значительный локальный магнитный момент

$$
2 M_{m}=(1+a)^{-1} \sum_{\alpha= \pm 1} \alpha \int_{-\infty}^{\infty} g_{\alpha, m}(\varepsilon) f(\varepsilon-\mu) d \varepsilon,
$$

который при малых значениях $\mathbf{M}_{\mathbf{q}_{0}, m}\left(\sim \mathbf{h}_{-\mathbf{q}_{0}}^{(D)}\right)$ следует трактовать как амплитуду нулевых флуктуаций (6). Наличие таковых позволяет согласовать экспериментальные данные по магнитным моментам с результатами расчетов.

3. В рассчитанной с учетом параметров $a b$ initio моделирования электронной структуре $\mathrm{MnSi}$, представленной на рис. 1 , величина хаббардовского отталкивания $U=0.93 \mathrm{eV}$, а параметр хундовского обменного взаимодействия $J=0.49 \mathrm{eV}$ [16]. Плотности состояний $d$-электронов, отвечающие разным значениям $m$, слабо зависят от $m$, т.е. $g_{0}^{(m, \sigma)}(\varepsilon) \approx g_{0}^{(\sigma)}(\varepsilon)$ 


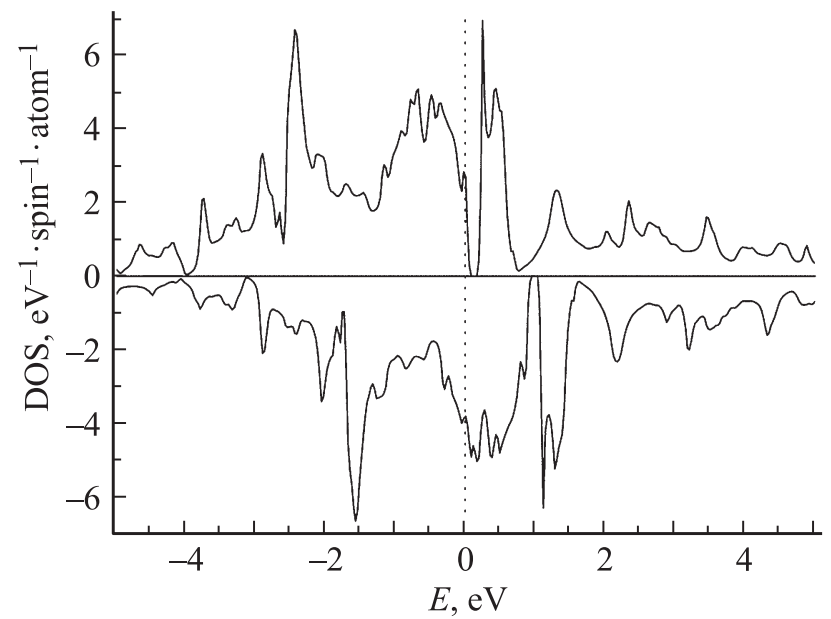

Рис. 1. Плотность симметризованных и антисимметризованных состояний при $T=0 \mathrm{~K}$. Плотность антисимметризованных состояний была умножена на минус единицу. Положение химического потенциала совпадает с началом отсчета энергии.

при $|\varepsilon-\mu| \leq U M^{(\mathrm{LDA})} \sim 1 \mathrm{eV}$. Поэтому, согласно уравнениям магнитного состояния и условиям перевала для функционала свободной энергии, намагниченность, амплитуда флуктуаций спиновой плотности электронов, среднеквадратичный магнитный момент на узле, a также функции плотностей спин-симметризованных и спин-антисимметризованных электронных состояний (как и определяемые через них другие величины) не зависят от квантового числа $m: \mathbf{M}_{\mathbf{q}_{0}, m} \equiv \mathbf{M}_{\mathbf{q}_{0}}$ и $M_{0, m} \equiv M_{0}$, $\left\langle M_{m}^{2}\right\rangle \equiv\left\langle M^{2}\right\rangle, \quad M_{m} \equiv M, \quad g_{\alpha, m}(\varepsilon) \equiv g_{\alpha}(\varepsilon) \quad\left(D_{m}^{-1} \equiv D^{-1}\right.$, $\chi_{\perp}^{(m)} \equiv \chi_{\perp}, \chi_{\|}^{(m)} \equiv \chi_{\|}, \kappa_{m} \equiv \kappa$ и т. д.).

Тогда в соответствии с (6) амплитуда тепловых спиновых флуктуаций пропорциональна квадрату температуры

$$
\begin{aligned}
\left\langle M^{2}\right\rangle_{T}= & 3 B(T / U)^{2}\left(q_{0} / k_{\mathrm{F}}\right)^{2}\left[\left(\lambda / R_{C}\right)^{2}-1+a A^{-1}\right]^{-1} \\
& \times\left[1+a A^{-1}+\left(q_{0} / k_{\mathrm{F}}\right)^{2}\left(\left(\lambda / R_{C}\right)^{2}-1\right)\right]^{-1}
\end{aligned}
$$

а амплитуда нулевых флуктуаций определяется выражением

$$
\begin{aligned}
\left\langle M^{2}\right\rangle_{0}= & \left(q_{0} / k_{\mathrm{F}}\right)^{2}\left(\pi^{2} A^{2} B U\right)^{-1} \sum_{\gamma}\left[\left(\lambda / R_{C}\right)^{2}-1\right] \\
& \times\left[1+\ln \left(1+A B^{-1}\left(R_{C} / \lambda\right)^{-2}\right)\right],
\end{aligned}
$$

где $R_{C}$ - радиус спиновых корреляций, определяемый выражением

$$
R_{C}=k_{\mathrm{F}}^{-1} A^{1 / 2}\left(D^{-1}+2 \kappa\left|M_{\mathbf{q}_{0}}^{(\gamma)}\right|+a\right)^{-1 / 2},
$$

$\lambda=2 \pi q_{0}^{-1}-$ период геликоидальной спирали.

Согласно термодинамическому условию минимума свободной энергии для относительного изменения объема, получаем

$$
\omega=\omega_{0 e}+\omega_{M},
$$

где

$$
\omega_{0 e}=\frac{1}{K W} \frac{5}{3} \sum_{a= \pm 1} \int g_{a}(\varepsilon)(\varepsilon-\mu) f(\varepsilon-\mu) d \varepsilon
$$

- вклад, связанный с одноэлектронными фермиевскими возбуждениями, спектр которых перенормирован спиновыми флуктуациями,

$$
\omega_{M}=\frac{U}{K W} \frac{5}{3} \chi_{\perp} M^{2}-\frac{U}{K W} \frac{5}{3}\left\langle M^{2}\right\rangle D^{-1}
$$

- магнитный вклад. В рассматриваемом случае полного вырождения по магнитному орбитальному квантовому числу $g_{a}(\varepsilon), M^{2}$ и $\left\langle M^{2}\right\rangle$ - плотность состояний, квадрат среднеквадратичного магнитного момента и квадрат амплитуды спиновых флуктуаций электронов всех пяти $d$-орбиталей.

Как показывает анализ спин-флуктуационных возбуждений и изменения локального момента на узле, наблюдаемые особенности термодинамических свойств в температурной окрестности магнитного фазового перехода связаны с резким уменышением радиуса спиновых корреляций при температуре $T_{S}$ и его последующим уменьшением в интервале от $T_{S}$ до $T_{C}[17,18]$. На рис. 2 представлены температурные зависимости радиуса спиновых корреляций, среднеквадратичного значения магнитного момента на узле $M$, намагниченности, рассчитанные в развитой модели электронной структуры с учетом уравнения магнитного состояния. Характерной особенностью результатов расчета является резкое уменьшение амплитуды нулевых флуктуаций (в соответствии с формулой $(11 \mathrm{~b}))$ вблизи температуры $T_{S}$. При этой температуре верхний край нижней подзоны симметризованных по спину $d$-состояний достигает положения химического потенциала (см. формулу (7)) вследствие роста

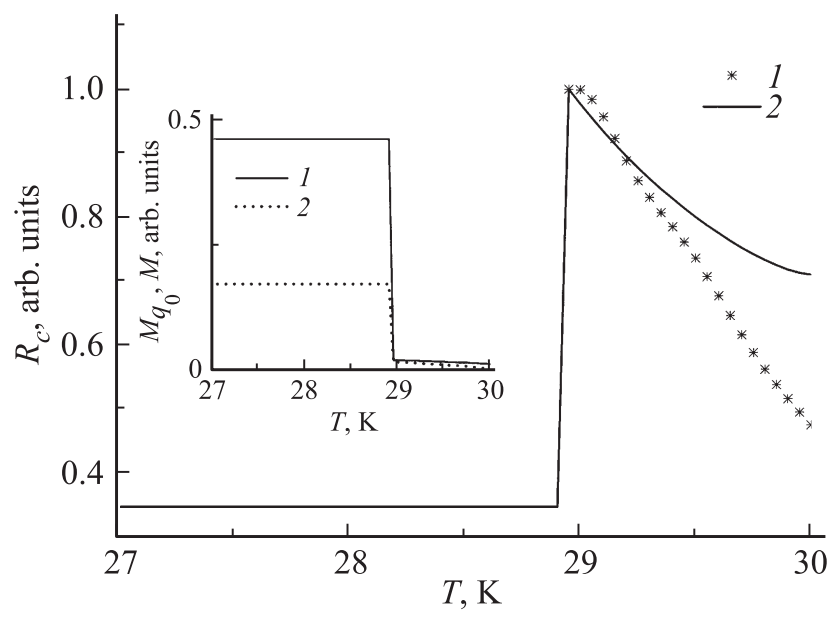

Рис. 2. Температурная зависимость радиуса корреляции при приближении к $T_{C} .1$ - экспериментальные данные [12], 2 - результаты расчета в настоящей работе по формуле (12). На вставке: 1 - температурная зависимость намагниченности, 2 - температурная зависимость магнитного момента. 


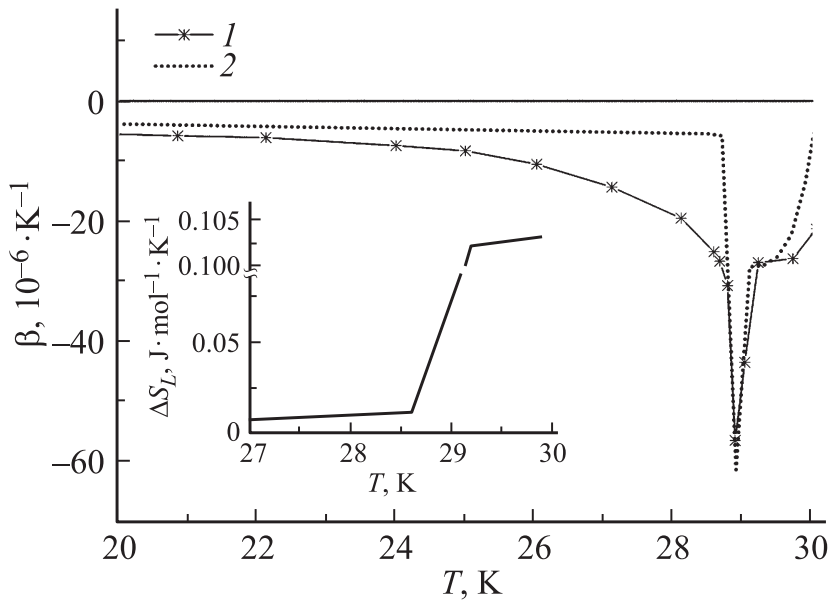

Рис. 3. Температурные зависимости электронного вклада $\beta$ в тепловое расширение $\mathrm{MnSi}$. 1 - экспериментальные данные [19], 2 - результат расчета $\beta(T)$ в настоящей работе. На вставке - изменение энтропии локальных магнитных моментов $\Delta S_{L}$.

амплитуды тепловых флуктуаций. В результате данная подзона оказывается полностью заполненной, и дальнейший рост среднеквадратичного магнитного момента с увеличением температуры оказывается невозможным. При этом в соответствии с (7) энергетический спектр антисимметризованных по спину $d$-состояний продолжает смещаться вверх по шкале энергий, что ведет к нарушению условия электронейтральности с увеличением температуры. В результате имеет место потеря термодинамической устойчивости всей электронной подсистемы, приводящая к исчезновению нулевых спиновых флуктуаций, перестройке спиновой подсистемы $d$-электронов и смене знака коэффициента $\kappa$ (возникновение неустойчивости ферромагнитных решений). Последнее приводит к возрастанию энтропии $S=(\partial F / \partial T)$ [16] и радиуса спиновых корреляций (рис. 2), а также сопровождается резким уменьшением объема (см. (13) и температурную зависимость КТР на рис. 3). При этом зависимостью самого магнитного момента и энергетического положения химического потенциала от изменения объема, как показывает численный анализ, можно пренебречь.

Дифференцируя выражение (4), с учетом (7) и условий перевала по объему и температуре находим объемный коэффициент теплового расширения

$$
\beta=\partial \omega / \partial T=\beta_{0 e}+\beta_{M} .
$$

Здесь

$$
\begin{aligned}
\beta_{0 e}= & -\frac{5}{3 K} T^{-1} \sum_{\beta= \pm 1} \int g_{0}(\varepsilon)(\varepsilon-\mu-\beta U m)^{2} \\
& \times f^{\prime}(\varepsilon-\mu-\beta U m) d \varepsilon
\end{aligned}
$$

- вклад фермиевских возбуждений, а $\beta_{M}$ - магнитный вклад, определяемый в магнитоупорядоченной области температур выражением

$$
\beta_{M}=2 \frac{U}{K} \frac{5}{3} \kappa\left(\frac{d M_{q_{0}}^{2}}{5 d T}\left\langle M^{2}\right\rangle+X_{q_{0}} \frac{d\left\langle M^{2}\right\rangle}{d T}\right),
$$

а в парамагнитной области температур

$$
\beta_{M}=\frac{U}{2 K} \frac{5}{3}\left(M \frac{d D^{-1}}{d M}+D^{-1}\right) \frac{d M^{2}}{d T},
$$

Расчет показывает, что на кривой КТР вблизи температуры $T_{S}$ возникает лямбда-подобная аномалия, выше которой на температурной зависимости формируется „Плечо“ (рис. 3). Аналогичная особенность формируется и на температурной зависимости намагниченности, которая выше температуры $T_{S}$ вследствие изменения знака параметра межмодовой связи $\kappa$ приближенно описывается выражениями

$$
\begin{gathered}
M_{q_{0}}=\left\langle M^{2}\right\rangle_{T_{C}}^{1 / 2}\left(1-\left(T / T_{C}\right)^{2}\right)^{1 / 2}, \\
T_{C}=\kappa^{-1} B^{-1} U\left(\left|D\left(\left\langle M^{2}\right\rangle_{T_{C}}^{1 / 2}\right)^{-1}\right|-a-d q_{0} / U\right)^{1 / 2},
\end{gathered}
$$

при этом значения радиуса корреляций спинов как в развитой модели, так и в эксперименте в противоречии с теорией переходов второго рода сохраняют конечные значения (рис. 2).

4. Таким образом исследование взаимосвязи электронной структуры и спинового состояния показывает, что магнитное состояние $\mathrm{MnSi}$ характеризуется двумя особенностями, возникающими вблизи температуры магнитного перехода, связанными с подавлением магнитных моментов геликоидального ферромагнитного состояния при $T_{S}$ и исчезновением геликоидального упорядочения при $T_{C}$. Следствием эффекта подавления нулевых флуктуаций являются резкое уменьшение радиуса спиновых корреляций, возрастание энтропии спиновой системы и отрицательный объемный эффект. В температурном интервале от $T_{S}$ до $T_{C}$ изменения электронной структуры, согласно (7), приводят к изменению знака параметра межмодовой связи в уравнениях магнитного состояния (9), (10), что делает ферромагнитное состояние неустойчивым и должно привести к флуктуациям направления оси квантования.

В том случае, когда уровень химического потенциала расположен в области энергий невырожденных орбитальных состояний, приходится учитывать эффект флуктуаций орбитальных магнитных моментов, который формально учтен в уравнениях (9), (10). Такая ситуация может возникнуть в сплавах $\mathrm{Fe}_{1-x} \mathrm{Co}_{x} \mathrm{Si}, \mathrm{Fe}_{1-y} \mathrm{Mn}_{y} \mathrm{Si}$, электронная структура которых требует отдельного изучения.

\section{Список литературы}

[1] S.V. Grigoriev, D. Chernyshov, V.A. Dyadkin, V. Dmitriev, E.V. Moskvin, D. Lamago, Th. Wolf, D. Menzel, J. Schoenes, S.V. Maleyev, H. Eckerlebe. Phys. Rev. B 81, 012408 (2010). 
[2] С.М. Стишов, А.Е. Перова. УФН 181, 1157 (2011).

[3] K.V. Shanavas, S. Satpathy. Phys. Rev. B 93, 195101 (2016).

[4] И.Е. Дзялошинский. ЖЭТФ 32, 1547 (1957).

[5] T. Moriya. Phys. Rev. 120, 91 (1960).

[6] P. Bak, M.H. Jensen. J. Phys. C 13, L881 (1980).

[7] О.Г. Страшников, А.А. Повзнер, П.В. Гельд. ФНТ 9, 1286 (1983).

[8] S.V. Grigoriev, S.V. Maleyev, A.I. Okorokov, Yu.O. Chetverikov, P. Böni, R. Georgii, D. Lamago, H. Eckerlebe, K. Pranzas. Phys. Rev. B 74, 214414 (2006).

[9] J.H. Wernick, G.K. Wertheim, R.C. Sherwood. Mater. Res. Bull. 7, 1431 (1972).

[10] M. Corti, F. Carbone, M. Filibian, T. Jarlborg, A.A. Nugroho, P. Carretta. Phys. Rev. B 75, 115111 (2007).

[11] T. Jeong, W.E. Pickett, Phys. Rev. B 70, 075114 (2004).

[12] M. Janoschek, M. Garst, A. Bauer, P. Krautscheid, R. Georgii, P. Böni, C. Pfleiderer. Phys. Rev. B 87, 134407 (2013).

[13] J.A. Hertz, M.A. Klenin. Phys. Rev. B 10, 1084 (1974).

[14] И.Е. Дзялошинский, П.С. Кондратенко. ЖЭТФ 70, 1987 (1976).

[15] V. Heine. Phys. Rev. 153, 673 (1967).

[16] А.А. Повзнер, А.Г. Волков, Т.А. Ноговицына. ФТТ 59, 211 (2017).

[17] А.А. Повзнер, А.Г. Волков, И.А. Ясюлевич. ФТТ 58, 1283 (2016).

[18] S.V. Grigoriev, S.V. Maleyev, E.V. Moskvin, V.A. Dyadkin, P. Fouquet, H. Eckerlebe. Phys. Rev. B 81, 144413 (2010).

[19] S.M. Stishov, A.E. Petrova, S. Khasanov, G.Kh. Panova, A.A. Shikov, J.C. Lashley, D. Wu, T.A. Lograsso. ЖЭТФ 133, 1017 (2008). 